\title{
Complete ligand loss in electron ionization of the weakly bound organometallic tungsten hexacarbonyl dimer
}

\author{
Michael Neustetter, ${ }^{a}$ Andreas Mauracher ${ }^{a}$, Paulo Limão-Vieira ${ }^{b^{*}}$ and Stephan Denifla ${ }^{a^{*}}$ \\ a. Institut für lonenphysik und Angewandte Physik, Leopold Franzens Universität Innsbruck, Technikerstrasse 25, A-6020 Innsbruck, Austria. \\ b. Laboratório de Colisões Atómicas e Moleculares, CEFITEC, Departamento de Física, Faculdade de Ciências e Tecnologia, Universidade NOVA de Lisboa, \\ 2829-516 Caparica, Portugal. \\ * Corresponding authors: Stehpan.Denifl@uibk.ac.at (Stephan Denifl); plimaovieira@fct.unl.pt (Paulo Limão-Vieira).
}

\begin{abstract}
We observed the bare $\mathrm{W}_{2}{ }^{+}$metal cation upon electron ionization of the weakly bound $\mathrm{W}(\mathrm{CO})_{6}$ dimer. This metal cation can be only observed due to fast conversion of the weak cluster bond into a strong covalent bond between the metal moieties.
\end{abstract}

The role of metal carbonyl complexes has been fully acknowledged in recent years in the field of plasma technology for metallic layers preparation ${ }^{1}$ as well as in nanotechnology to deliver metallic structures. ${ }^{2,3}$ Among the species, tungsten hexacarbonyl $\mathrm{W}(\mathrm{CO})_{6}$, is one of the most important because of its chemical properties well-attuned to prepare tungsten layers and tungsten interconnection in Electron Beam Induced Deposition (EBID) and Ion Beam Induced Deposition (IBID) techniques. ${ }^{4-7}$ As so, in these high-energy (tens of keV) electronand ion-driven processes, low-energy secondary electrons (typically $<100 \mathrm{eV}$ ) are produced by the interaction of the primary beam with the surface and surface deposits, which can trigger efficient dissociative ionization and electron attachment reactions to deposited organometallic molecules on the surface of a solid substrate. The decomposition process of $\mathrm{W}(\mathrm{CO})_{6}$ is then triggered via CO cleavage with the volatile materials pumped out. However, these secondary electron interactions can efficiently determine the nature of deposits formed on the surface and are responsible to cause significant deterioration of the spatial resolution of these techniques. ${ }^{8}$

EBID and/or FEBIP (Focused Electron Beam Induced Processing) techniques, have been used to create structurally well-defined, metal-containing nanostructures capable of sub-10 nm size (e.g. $1.5 \mathrm{~nm}$ nano-dots) $)^{9,10}$, in detriment to the traditional nanotechnology processing techniques that mainly rely on resist layers which limits their spatial control and resolution. In FEBID, a tightly focused high-energy electron beam (spot sizes of $\sim 0.2 \mathrm{~nm}$ ) impinges into a surface exposed to a continuous stream of precursor molecules (typically $\sim 0.7 \mathrm{~nm}$ width) ${ }^{11}$ and through electron-molecule interactions these decompose to form, in the ideal case, a chemically and structurally welldefined (e.g. pure metal) deposit while volatile fragments are pumped away (see e.g. Ref. [12]). Such level of spatial resolution is extremely relevant for fabrication of functional nanomaterials due to increasing demand for even-smaller structures with properties tailored to specific applications, control over the deposits composition, reduce broadening, and increase deposition rate.

Several gas-phase studies on electron interactions with isolated EBID precursor molecules $\mathrm{W}(\mathrm{CO})_{6}, \mathrm{Co}(\mathrm{CO})_{3}(\mathrm{NO}), \mathrm{MeCpPtMe}_{3}$ and $\mathrm{Pt}\left(\mathrm{PF}_{3}\right)_{4}$, have been reported in the literature ${ }^{13-17}$, where dissociative electron attachment (DEA) has been suggested to be an important decomposition process lending support to bond breaking of the same molecules adsorbed on solid surfaces when exposed to $500 \mathrm{eV}$ electrons..$^{18}$ Of relevance, DEA leads to incomplete loss of CO ligands, conferring therefore a particular contaminating behaviour on the edge of the deposit. ${ }^{16,18}$ Notwithstanding, gas-phase electron ionization of $\mathrm{W}(\mathrm{CO})_{6}$ leads to $\mathrm{W}^{+}$formation above a threshold at $\sim 21 \mathrm{eV}^{15}$, showing effective $C O$ ligand cleavage, which may result in a more efficient detriment than DEA in the EBID technique.

In order to bridge a gap between the dissociation processes of single isolated molecules in the gas-phase and the actual surface experiments, which are certainly very relevant within the context of EBID and IBID techniques, there is an urgent need to shed light on the underlying molecular processes in clusters of tungsten hexacarbonyl ionized by electron ionization. To our knowledge no direct mass spectrometric observation of cations formed upon electron ionization of Van-der-Waals bonded $\mathrm{W}(\mathrm{CO})_{6}$ aggregates has been reported yet. As so, we have performed investigations on such cation formation in order to give a detailed description of the processes initiated by $70 \mathrm{eV}$ electrons in bare $\mathrm{W}(\mathrm{CO})_{6}$ aggregates. Within these molecular structures, $\mathrm{W}(\mathrm{CO})_{6}$ complexes (here as aggregates fully stabilized in the intracluster environment prior to electron interaction) may lead to ligands removal upon the electron ionization process as observed for the single molecule. However, the environment may stabilize a particular dissociation channel or generally buffer dissociation as well due to the energy dissipative environment. In the course of our present experiments we observed, among a rich fragmentation pattern, the bare $\mathrm{W}_{2}^{+}$ion, which is formed by loss of 12 covalently bound $\mathrm{CO}$ ligands from the dimer. In the following we 
find explanations how the intermolecular bond will sustain such strong fragmentation process.

The experiments were performed in a crossed electron-cluster beam experiment equipped with a double focusing mass spectrometer, which has been described in detail before. ${ }^{19}$ Cations formed in a Nier type ion source were accelerated by 6 $\mathrm{kV}$, analysed by their momentum and energy in a magnetic and electric sector, respectively, and finally detected by a channeltron type secondary electron multiplier. The ion source was providing an electron beam with a resolution of $\sim 1 \mathrm{eV}$ and beam currents of $10 \mu \mathrm{A}$ used for the ionization measurements. The electron energy scale was calibrated with respect to the formation of $\mathrm{Ar}^{+} / \mathrm{Ar}$ at $15.8 \mathrm{eV}$ and $\mathrm{Ar}^{2+} / \mathrm{Ar}$ at $43.4 \mathrm{eV} .{ }^{20} \mathrm{~A}$ neutral cluster beam of $\mathrm{W}(\mathrm{CO})_{6}$ was formed by supersonic expansion through a $50 \mu \mathrm{m}$ orifice kept at $150{ }^{\circ} \mathrm{C}$ to avoid clogging. For better clustering conditions the solid tungsten hexacarbonyl sample (Sigma Aldrich, Vienna, stated purity $\geq 97$ $\%)$ was heated up to about $90^{\circ} \mathrm{C}$ and argon was used as seeding gas ( 0.5 bar). Mass spectra were recorded up to the trimer. Very low ion intensity above the dimer (see Figure S1, ESI $†$ ) indicates that under our expansion conditions the formation of clusters larger than the dimer is unfavourable. The cluster and ionization chambers were separated by a skimmer with $1 \mathrm{~mm}$ opening, with working pressures of $\sim 4 \times 10^{-4} \mathrm{mbar}$ in the former and $4 \times 10^{-}$ ${ }^{6}$ mbar in the latter. A moveable plate inside the ion source chamber enables to measure the background which contributes to the monomer. The background arising from monomers has been subtracted from all measurements presented here. In order to assign the contributing cluster fragments, we have obtained from the mass spectra contributions from the natural $\mathrm{W}$ isotope distribution: ${ }^{182} \mathrm{~W} 27.7 \%,{ }^{183} \mathrm{~W} 15.1 \%,{ }^{184} \mathrm{~W} 32.3 \%$, and ${ }^{186} \mathrm{~W} 24.9 \%$, fitted to the observed intensities.

In order to interpret the experimental results shown in the following we performed quantum chemical calculations using the hybrid density functional M06-2X with the basis sets 6$311+G(3 d f)$ for second row elements and LanL2TZ(f) for the metal atoms. The fully optimized structure of the neutral $\mathrm{W}(\mathrm{CO})_{6}$ dimer is shown in Figure 1 . Thereby the molecules arrange rotated to each other. The intermolecular bond length ranges between $3.317 \AA(\mathrm{O} \cdots \mathrm{O})$ and $5.488 \AA$ (the central W...W bond). The binding energy of the neutral is $0.24 \mathrm{eV}(0.23 \mathrm{eV}$ including zero point correction) and has the magnitude of a Vander-Waals bond.

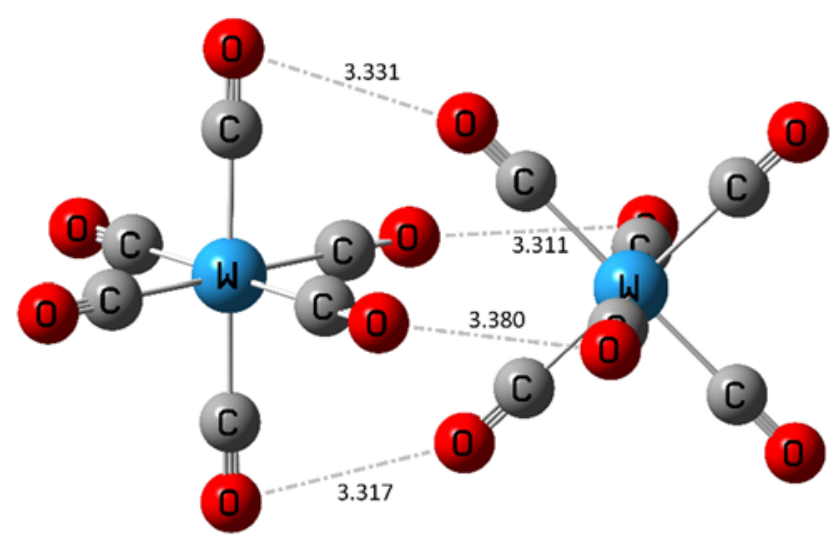

Figure 1. Optimized structure of the tungsten hexacarbonyl dimer obtained using the hybrid density functional M06-2X with the basis sets $6-311+G$ (3df) (for $C$ and $\mathrm{O}$ atoms) and LanL2TZ(f) (for W), respectively. The distances shown are given in Ångström.

Upon electron ionization the dimer breaks up and mainly fragment ions can be observed in the electron ionization mass spectrum at the electron energy of $70 \mathrm{eV}$. For example the amount of the dimer ion is about $27 \%$ of the most abundant cluster fragment $\mathrm{W}_{2}(\mathrm{CO})_{9}{ }^{+}$(not shown). Monomer ionization mainly involves loss of $n$ (CO) ligands ${ }^{15}$, which is also a characteristic fragmentation after the ionization of the dimer. In the following we focus on the product ions formed after substantial loss of ligands. Thus, only the section of the mass spectrum from the monomer (molecular weight $351.90 \mathrm{u}$ ) up to about $386 \mathrm{u}$ is shown in Figure 2.

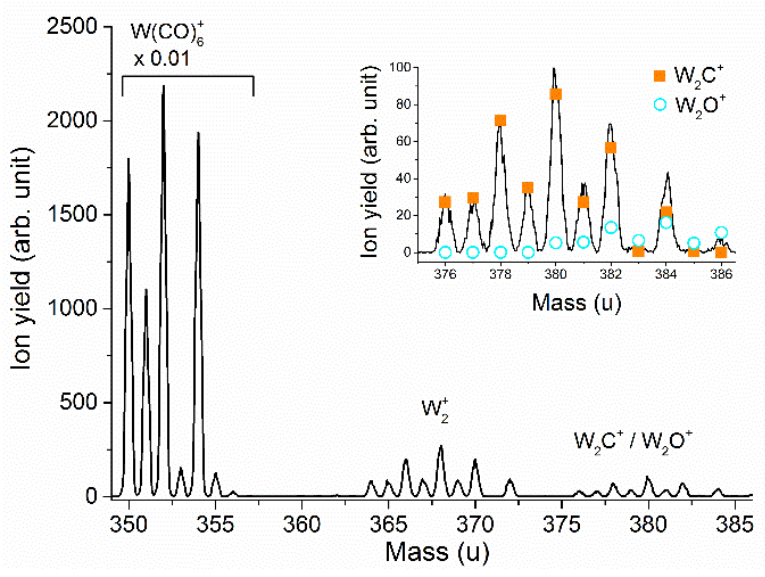

Figure 2. Electron ionization mass spectrum in the region of the monomer ion $\mathrm{W}(\mathrm{CO})_{6}{ }^{+}$up to about $386 \mathrm{u}$. The spectrum shown was recorded at the electron energy of $70 \mathrm{eV}$. The inset shows a detailed view of the ion yield abundance in the region between $376 \mathrm{u}$ and $386 \mathrm{u}$ which is a superposition of $\mathrm{W}_{2} \mathrm{C}^{+}$(filled squares) and $\mathrm{W}_{2} \mathrm{O}^{+}$(open circles).

We note that, although visible in Figure 2 with a modest contribution relative to the intense ion $\mathrm{W}(\mathrm{CO})_{6}{ }^{+}, \mathrm{W}_{2}{ }^{+}$formation can be observed which lends support to the efficient clustering environment to stabilize its formation free of any CO ligand. This is a remarkable finding; how a weak intermolecular bond obviously could survive the complete ligand removal from both moieties? We note that previously a similar observation was made in the case of electron ionization of $\mathrm{SF}_{6}$ clusters. ${ }^{21}$ In this case the formation of $\mathrm{S}_{2} \mathrm{~F}_{5}{ }^{+}$was reported, i.e. apparent molecular fragmentation of two weakly bond cluster moieties occurred; though not complete as observed for the present organometallic dimer. The authors in Ref. [21] claimed that such 
dissociation process cannot occur in a conventional electron ionization process of a cluster and instead they ascribed it to an ion-molecule reaction or a sequential electron ionization process. ${ }^{21}$ In order to rule out any secondary process or impurity (for example clustering of decomposed tungsten hexacarbonyl) we determined the ion efficiency curve of $\mathrm{W}_{2}{ }^{+}$close to the ionization threshold. The corresponding measurement and a fit based on the Wannier threshold $\operatorname{law}^{22}$ is shown in Figure 3 together with that of $\mathrm{W}_{2} \mathrm{C}^{+}$for comparison. We derived a threshold of $29.3 \pm 0.6 \mathrm{eV}$ for $\mathrm{W}_{2}^{+}$and $32.9 \pm 0.6 \mathrm{eV}$ for $\mathrm{W}_{2} \mathrm{C}^{+}$ which is formed by cleavage of the strong $\mathrm{C}-\mathrm{O}$ bond ( 11.2 eV). ${ }^{15}$
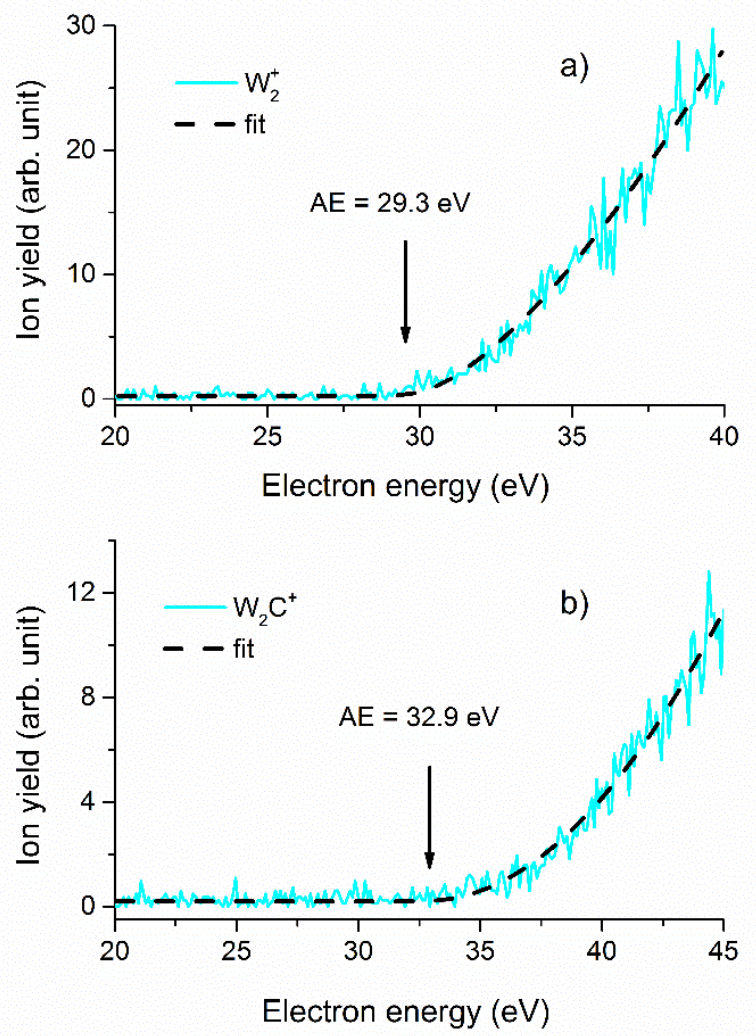

Figure 3. $\mathrm{W}_{2}{ }^{+}$ion yield (a) and $\mathrm{W}_{2} \mathrm{C}^{+}$ion yield (b) as a function of the initial electron energy. The experimental data are shown as solid lines, while the fits based on the Wannier threshold law are shown in dashed lines.

In assertion of the experimental data, quantum chemical calculations were performed for the reactions yielding these ions. For $\mathrm{W}_{2}^{+}$, the reaction can be schematically represented as $\left[\mathrm{W}(\mathrm{CO})_{6}\right]_{2}+\mathrm{e}^{-} \rightarrow \mathrm{W}_{2}^{+}+12(\mathrm{CO})$ with a reaction enthalpy of 25.97 $\mathrm{eV}$. This energy is in reasonable accord with the experimental value of $29.3 \mathrm{eV}$. The deviation can be explained by the nature of the dissociative electron ionization process where the experimental onset not necessarily matches the reaction enthalpy due the vertical electronic transition from the neutral state to an excited ionic state at equilibrium distance of the neutral. In addition, energy barriers which may occur along the reaction coordinate are not taken into account. We note that the thresholds of fragment ions formed upon ionization of the monomer have substantially lower thresholds(see Table 1), i.e., a secondary reaction in which e.g. a $\mathrm{W}^{+}$reacts with a neutral $\mathrm{W}$ can be ruled out. This is due to the required energy of about $13.1 \mathrm{eV}$ for the reaction $\mathrm{W}(\mathrm{CO})_{6} \rightarrow \mathrm{W}+6(\mathrm{CO})$ which is thus far below the observed threshold of $\mathrm{W}_{2}^{+}$. In addition, we also rule out a secondary reaction of the ionized dimer, $\mathrm{W}_{2}(\mathrm{CO})_{12}{ }^{+}$, which is already formed close to $12 \mathrm{eV}$ (see Figure S2, ESI $\dagger$ ).

Table 1: Appearance energies $(\mathrm{AE})$ of $\mathrm{W}(\mathrm{CO})_{i}{ }^{+} \mathrm{i}=0-6$, data taken from Ref. [15]

\begin{tabular}{|l|l|}
\hline Ion & $\mathrm{AE}(\mathrm{eV})^{15}$ \\
\hline $\mathrm{W}(\mathrm{CO})_{6}{ }^{+}$ & $8.47 \pm 0.06$ \\
\hline$W(\mathrm{CO})_{5}{ }^{+}$ & $10.31 \pm 0.06$ \\
\hline$W(\mathrm{CO})_{4}{ }^{+}$ & $12.01 \pm 0.06$ \\
\hline$W(\mathrm{CO})_{3}{ }^{+}$ & $13.91 \pm 0.06$ \\
\hline$W(\mathrm{CO})_{2}{ }^{+}$ & $16.43 \pm 0.06$ \\
\hline$W(\mathrm{CO})^{+}$ & $18.35 \pm 0.06$ \\
\hline$W^{+}$ & $20.87 \pm 0.06$ \\
\hline
\end{tabular}

Instead one can observe a noteworthy change of the energetics when going from the monomer to the dimer. In the case of electron ionization of single tungsten hexacarbonyl, the experimental threshold for formation of $\mathrm{W}^{+}$was $\sim 21 \mathrm{eV}$ (Table 1), i.e. about $8.3 \mathrm{eV}$ lower than in the present case for $\mathrm{W}_{2}{ }^{+}$. Comparing the latter value with the mean bond dissociation energy of a carbonyl ligand $(\sim 2 \mathrm{eV})$, the expected threshold value would be $\sim 12 \mathrm{eV}$ higher than that for $\mathrm{W}^{+}$. Hence the complete decarbonylation becomes energetically more favourable in the dimer compared to the monomer. The present calculations indicate that this effect results from the conversion of the initially weak Van-der-Waals bond between the tungsten atoms to a strong covalent bond in the final $\mathrm{W}_{2}{ }^{+}$configuration (bond energy $7.50 \mathrm{eV}$ at internuclear distance of $3.317 \AA$ ). . We note that previous one-color femtosecond pump/probe experiments with neutral chromium hexacarbonyl clusters indicated ultrafast metal-metal bond formation after excitation and formation of bare $\mathrm{Cr}_{n}{ }^{+}$by the ionizing probe pulse. ${ }^{23}$ From these results and although we are not aware of any ultrafast photon experiment on tungsten hexacarbonyl, we may assume that ultrafast dynamics must also proceed in $\mathrm{W}(\mathrm{CO})_{6}$ since the van-der Waals bond between the metal atoms would break during the hard fragmentation process typical for electron ionization. However, to confirm this hypothesis, detailed theoretical studies on the intermediate transition states would be necessary which are beyond the scope of the present work. In order to deduce implications of the present findings for EBID and IBID techniques we note that recent surface science studies of $500 \mathrm{eV}$ incident electrons with adsorbed $\mathrm{W}(\mathrm{CO})_{6}$ molecules, $\mathrm{X}$-ray photoelectron spectroscopy, mass spectrometry and infrared spectroscopy revealed that electron stimulated desorption leads to multiple CO ligand breaking. ${ }^{18}$ As so, such process yields partially decarbonylated tungsten species assigned to $W_{x}(\mathrm{CO})_{y}$ rather than $W(C O)_{y}$ on the basis of ability 
of metal carbonyls to form polynuclear metal carbonyl clusters. However, such evidence was not experimentally confirmed in these condensed phase structures ${ }^{18}$, but clearly visible here in the ion yields of Figure 2 and 3 . Of relevance is the fact that the surface studies also report the ability of electron interactions with $\mathrm{W}_{\mathrm{x}}(\mathrm{CO})_{\mathrm{y}}$ species to produce ligand decomposition in detriment to further $\mathrm{CO}$ desorption, which certainly has relevance in producing oxidized tungsten atoms incorporated in a carbonaceous matrix. ${ }^{18}$ In the present mass spectrum we observe indeed a small amount of the oxidized tungsten dimers, i.e. $\mathrm{W}_{2} \mathrm{O}^{+}$in accordance with the observations of surface studies. Such remarkable rearrangement reactions were reported for the single molecule upon electron ionization. ${ }^{15}$ According to our results, these reactions remain also operative for agglomerates.

In conclusion, the present study reports electron ionization of $\mathrm{W}(\mathrm{CO})_{6}$ dimer, which provides a reaction environment which complexity bridges the simple gas-phase and condensed phase studies. We observe strong fragmentation reactions up to the loss of all $\mathrm{CO}$ ligands from both tungsten hexacarbonyl moieties, i.e. formation of $\mathrm{W}_{2}{ }^{+}$. Such process of the metal dimer formation becomes only possible due to fast conversion of the initial weak Van-der-Waals bonds between the two moieties to strong covalent bonds between the metal atoms. The quantum chemical calculations predict a binding energy of $6.87 \mathrm{eV}$ for the neutral against $7.50 \mathrm{eV}$ for the cation. The latter is also responsible for a noteworthy lowering of the threshold for the bare metal cation, in comparison to the single molecule. The present studies demonstrate the possibility that the bare metal dimer cation can be produced in a single electron interaction, which will contribute to the underlying processes in EBID and IBID techniques. However, the relative abundance of this ion is minor and thus not a significant pathway in the production of bare metal deposits on surfaces. This result is in line with the limited spatial resolution observed in surface experiments.

\section{Acknowledgments}

This work has been supported by FWF, Wien, Austria (I1015). PLV acknowledges funding from the Portuguese Foundation for Science and Technology (FCT-MEC) through research grants PTDC/FIS-ATO/1832/2012 and UID/FIS/00068/2013 and the EU/ESF COST Action CELINA - CM1301 for some part of this work.

\section{References}

1 K. K. Lai, H. H. Lamb, Thin Solid Films, 2000, 370, 114.

2 C. W. Hagen, W. F. van Dorp, P. A. Crozier, P. Kruit, Surf. Sci., 2008, 602, 3212.

3 K. E. Lewis, D. M. Golden, G. P. Smith, J. Am. Chem. Soc., 1984, 106, 3905.

4 I. J. Luxmore, I. M. Ross, A. G. Cullis, P. W. Fry, J. Orr, P. D. Buckle, Thin Solid Films, 2007, 515, 6791.

5 M. Song, K. Furuya, Sci. Technol. Adv. Mater., 2008, 9, 023002.

6 F.Porrati, R. Sachser, M. Huth, Nanotechnology, 2009, 20, 195301.
7 I. Sychugov, Y. Nakayama, K. Mitsuishi, J. Phys. Chem. C, 2009, 113, 21516.

8 N. Silvis-Cividjian, C. W. Hagen, L. H. A. Leunissen, P. Kruit, Microelectron. Eng., 2002, 61-62, 693-699.

9 M. Tanaka, M. Shimojo, M. Han, K. Mitsuishi, K. Furuya, Surf. Interface Anal., 2005, 37, 261.

10 W. F. van Dorp, C. W. Hagen, P. A. Crozier, P. Kruit, Nanotechnology, 2008, 19, 225305.

11 W. F. van Dorp, I. Lazic, A. Beyer, A. Gölzhäuser, J. B. Wagner, T. W. Hansen, C. W. Hagen, Nanotechnology, 2011, 22, 115303.

12 W. F. van Dorp, C. W. Hagen, J. Appl. Phys., 2008, 104, 081301.

13 S. Engmann, M. Stano, Š. Matejčik, O. Ingólfsson, Phys. Chem. Chem. Phys., 2012, 14, 14611.

14 S. Engmann, M. Stano, Š. Matejčik, O. Ingólfsson, Angew. Chem. Int. Ed., 2011, 50, 9475.

15 K. Wnorowski, M. Stano, W. Barszczewska, A. Jówko, Š. Matejčik, Int. J. Mass Spectrom., 2012, 314, 42.

16 K. Wnorowski, M. Stano, C. Matias, S. Denifl, W. Barszczewska, Š. Matejčik, Rapid Commun. Mass Spectrom., 2012, 26, 2093.

17 O. May, D. Kubala, M. Allan, Phys. Chem. Chem. Phys., 2012, 14, 2979.

18 S. G. Rosenberg, M. Barclay, D. H. Fairbrother, Phys. Chem. Chem. Phys., 2013, 15, 4002.

19 D. Almeida, D. Kinzel, F. F. da Silva, B. Puschnigg, D. Gschliesser, P. Scheier, S. Denifl, G. García, L. González, P. Limão-Vieira, Phys. Chem. Chem. Phys., 2013, 15, 11431.

20 B. Gstir et al., J. Phys. B, 2002, 35, 2993-3007.

21 A. Stamatovic, P. Scheier, T. D. Märk, J. Chem. Phys., 1988, 88, 6884 .

22 M. M. Dawley, K. Tanzer, W. A. Cantrell, P. Plattner, N. R. Brinkmann, P. Scheier, S. Denifl and S. Ptasińska, Phys. Chem. Chem. Phys., 2014, 16, 25039-25053.

23 M. Gutmann, M.S. Dickebohm and J. M. Janello, J. Phys. Chem. A, 1999, 103, 2580-2591. 